Controlled clinical balance studies of the effects of anticonvulsant drugs and the ketogenic diet (KD) on acid-base, electrolyte, and amino acid metabolism in children with absence seizures (Millichap JG et al. Epilepsia 1964;5:239-255; Am J Dis Children 1964;107:593-604) found that the KD and acetazolamide, studied as monotherapies, had similar metabolic effects. They both caused a metabolic acidosis, with decreased $\mathrm{pH}, \mathrm{pCO} 2$, and standard bicarbonate, and a negative balance of electrolytes. In contrast, trimethadione, mephobarbital, and methsuximide as monotherapies (anti-'petit mal' medications available in the 1960s), caused a metabolic alkalosis, with an elevation of $\mathrm{pH}$ and standard bicarbonate, and compensatory rise in $\mathrm{pCO}$; the urinary excretion of sodium and potassium and fecal excretion of calcium, magnesium and phosphorus were reduced, and the balance of electrolytes was positive. The effects of the ketogenic diet and acetazolamide on acid-base and electrolyte balance were the reverse of those obtained during treatment with conventional antiepileptic medications and corticotropin. Apart from an increase in serum leucine during treatment with the KD, levels of serum amino acids showed no significant changes. In this study, while all therapies were beneficial, the KD was most effective in the control of absence seizures and reduction of epileptiform discharges in the EEG. Concurrent use of a carbonic anhydrase inhibitor (acetazolamide or zonisamide) and KD would result in accentuated effects on acid-base and electrolyte metabolism and potential improvement in seizure control, but not without an anticipated increase in adverse side effects.

\title{
QUANTITATIVE EEG, COGNITIVE DEFICITS, AND BECTS
}

Researchers at Pontificia Universidade Catolica, Campinas, Brazil studied the relationship between educational problems and clinical/EEG aspects of benign childhood epilepsy with centrotemporal spikes (BECTS) in 38 children, ages 8 to 11 years (average age 9.29 +/ 1.27). Educational problems assessed by the School Performance Test, Parent and Teacher Questionnaires on learning difficulties, and the WISC-III test were observed in 7 (18.4\%) children with BECTS. In this subgroup of educationally handicapped children, relative alpha amplitudes at the central and parietal electrodes were lower as compared with the BECT subgroup with normal educational performance and a control group matched for age and gender. Alterations in background brain electrical activity appeared to be related to a tendency toward educational disorders in children with BECTS. Quantitative EEG is a possible means of assessment of cognitive deficits in children with BECTS. (Tedrus GMAS, Fonseca LC, Melo EMV, Ximenes VL. Educational problems related to quatitative EEG changes in benign childhood epilepsy with centrotemporal spikes. Epilepsy \& Behav Aug 2009;15:486-490). (Respond: Dr Lineu C Fonseca, Dept of Neurology, Pontificia Universidade Catolica de Campinas, Brazil. E-mail: lineu.fonseca@uol.com.br).

COMMENT. Deonna T, Roulet E and associates, of Lausanne, Switzerland, in one of the earlier prospective neuropsychological and EEG studies of 22 children with BECTS (19) and occipital spikes (3), found 21 had average IQ, 8 had school difficulties, 4 delayed language development, and 8 had transient impairments in verbal, visuospatial, or memory function. Cognitive deficits improved or normalized on follow-up, with concomitant EEG improvement or normalizaton. Transient cognitive difficulties in some children with BECTS were directly related to the paroxysmal EEG activity (Deonna $T$ et al. Dev Med Child Neurol 2000;42:595-603). Wolff M et al (Epilepsia 2005;46:1661-1667), in a combined 
MEG/EEG study of children with benign partial epilepsy, reported a correlation between location of focal interictal spikes and selective cognitive deficits. Massa R et al (Neurology 2001;57:1071-1079) found that EEG interictal patterns and their persistence were the hallmarks of neuropsychological impairments in 10 of 35 patients $(28 \%)$ with BECTS followed from onset to complete recovery. While these studies focused on the correlation between epileptiform discharges and cognitive impairments in children with BECTS, the current study from Brazil notes a relation between alterations in amplitude of background EEG activity and educational problems.

Ictal EEG and BECTS. Tedrus et al, the Brazil research team, also report details of an ictal EEG during oropharyngeal seizures in an 8-year-old boy with BECTS (Clinical EEG and Neuroscience July 2009;40(3):200-203). Seizure onset during sleep correlated with an increase in centrotemporal spikes followed by slow waves in the right hemisphere. A brief decrease in amplitude of background activity preceded rhythmic, diffuse sharp waves in the right centrotemporal region. Finally, high potential spikes reappeared in the central and temporal regions of the right hemisphere with normalization of background activity. The EEG changes occurred concurrently with clonic lip movements, pouting, and throat noises.

\section{COGNITIVE DEFICITS AT ONSET OF EPILEPSY}

Neuropsychological functioning and academic achievement were determined at the time of the first seizure in a prospective study of 282 children (ages 6-14 years, with IQ > 70) and compared with 147 healthy siblings examined at Indiana University, Case Medical Center, and Cincinnati Children's Hospital. Children with seizures scored lower than siblings on all neuropsychological tests, especially involving attention/executive/construction, but academic achievement was unaffected. Neuropsychological deficit was exhibited by $27.4 \%$ of children with seizures vs $18.2 \%$ of healthy siblings $(\mathrm{p}=0.04)$. Almost twice as many with seizures showed deficits in attention/executive/construction, verbal memory and learning, language and processing speed. Only symptomatic/cryptogenic seizure etiology was a risk factor for cognitive deficit. Children taking valproic acid scored lower for processing speed vs no-AED group $(\mathrm{p}=0.009)$. Among children with seizures, those on AEDs performed worse than no-AED group on all neuropsychological factors, but not on academic achievement. EEG epileptiform activity was associated with slower processing speed $(\mathrm{p}=0.004)$ but not with academic achievement. Risk factors for cognitive deficits were multiple seizures, AEDs, symptomatic/cryptogenic etiology, and epileptiform activity on initial EEG; a child with all 4 risk factors is 3 times more likely than healthy siblings to have neuropsychological deficits by the first clinic visit. Absence epilepsy was an added risk factor. (Fastenau PS, Johnson CS, Perkins SM, et al. Neuropsychological status at seizure onset in children. Risk factors for early cognitive deficits. Neurology Aug 12, 2009;73:526-534). (Respond: Philip S Fastenau $\mathrm{PhD}$, Department of Neurology, University Hospitals Case Medical Center, 11100 Euclid Ave, HAN 5040, Cleveland. OH 44106. E-mail: Philip.Fastenau@uhhospitals.org).

COMMENT. Children identified with a first seizure exhibit neuropsychological deficits in $27 \%$ cases. In those with risk factors, up to $40 \%$ have cognitive deficits. Children with multiple seizures, symptomatic/cryptogenic seizure etiology, those on AEDs, or with epileptiform discharges on the initial EEG should be referred for neuropsychological and educational evaluation. 